\title{
Can the gag reflex be controlled by acupuncture?
}

\author{
The role of acupuncture in controlling the gagging reflex using a review of ten cases J. Fiske and C. Dickinson \\ Br Dent J 2001; 190: 611-613
}

\begin{abstract}
Aim
The gagging reflex is a physiological reaction which safeguards the airway from foreign bodies. In some people this response is exaggerated to the extent that the acceptance/provision of dental treatment is not possible. The aim of this paper is to review the role of acupuncture in controlling gagging as a safe, cheap, quick and relatively non-invasive technique.
\end{abstract}

\section{Method \\ 10 people agreed to try ear acupuncture to control gagging during dental treatment. Prior to treatment the severity of gagging was assessed. Acupuncture needles were inserted into a specific anti-gagging point on each ear, manipulated briefly and left in situ. Dental treatment was then carried out and the effectiveness of the acupuncture in preventing gagging was assessed. After treatment, the needles were removed and the patient discharged. All acupuncture was carried out by a dentist trained in its use.}

\section{Results}

Four people had a severe gag reflex which made treatment impossible and six had a very severe reflex which made treatment impossible and affected their dental attendance. Ear acupuncture completely controlled the gag reflex in eight cases ( 23 treatment episodes) and partially controlled the reflex in two cases (two treatment episodes). Dental treatment could be carried out in all cases and at all visits. The cost of materials was $\mathfrak{E} 0.2$ per person per visit. Additional clinical time was in the order of $2-3$ minutes. There were no adverse reactions to the technique and, on all occasions, patients were fit to leave the surgery and travel home unaccompanied.

\section{Conclusions}

Ear acupuncture was successful in controlling the gag reflex. It is a safe, quick, inexpensive and relatively non-invasive technique. A controlled clinical trial is required to investigate any placebo effect.

\section{In Brief}

- The aetiology of gagging is complex and not fully understood.

- Gagging can become a conditioned response that makes dental treatment difficult or impossible for both the individual and the dentist. It can result in the avoidance of dental care.

- Ear acupuncture is a simple, cheap and relatively non-invasive technique that can be useful in controlling the gag reflex.

- The placebo effect of the technique needs to be investigated before its effectiveness is fully known.

\section{Comment}

A cupuncture is a technique for medical $A$ treatment first formally described about 2,600BC in China by the Emperor Hung $\mathrm{Ti}$, although there is evidence of use prior to this. A range of different acupuncture techniques have evolved, with some involving the whole body, some involving specific areas, such as the ear, or hand. This paper looks at the use of a specific ear acupuncture technique in the management of patients with a prominent retch reflex, a problem familiar to all practitioners, which can be so severe as to prevent dental treatment, or inhibit patients from seeking treatment.

In the paper a small group of patients with documented severe retch problems were offered ear acupuncture as a management technique during their treatment episodes, with quantification of the problems and improvement in the retch reflex during treatment. The paper reports a consistently good response to the use of acupuncture in the control of the retch reflex for all the patients in the group, with all patients being able to receive treatment, although some had been unable to accept treatment in the past, and others only with IV sedation. It is interesting to note that most of the patients in the group received multiple exposures to treatment with the management of the retch reflex by acupuncture, with effective control, and by implication satisfactory working conditions, indeed the authors indicate a wide range of treatments. Whilst the placebo effect is impossible to eliminate in such circumstances, as this not a controlled study, the reproducibility reported does add to this paper. The authors, who are reporting on a series of clinical cases that show an association, highlight the potential role of the placebo.

In comparison with other methods for management of the retch reflex the authors show the technique to be cheap, quick, and with no morbidity, in contrast to sedation using Midazolam. When we strive for the treatments with the best results and least morbidity such an approach would appear to have much to commend it.

\section{Tom Thayer}

Senior Dental Officer \& Specialist in Surgical Dentistry, Chester \& Halton

Community NHS Trust 